\title{
A colaboração científica nas áreas de informação no Brasil
}

\section{(1972-2013)}

\author{
Jayme Leiro Vilan Filho \\ jleiro@unb.br \\ Universidade de Brasília, Faculdade de Ciência da Informação, Brasília, DF, Brasil
}

Resumo: A colaboração entre cientistas é um indicador relacionado com a produção científica e normalmente ambos podem ser obtidos de bases de dados bibliográficos usadas como fontes de informação para tomada de decisão de gestores de Ciência, Tecnologia \& Inovação, autores, editores, cientistas e estudantes. Estudos anteriores proporcionaram uma visão da produção e da colaboração científica brasileira nas áreas de informação até 2010 e o presente estudo atualiza as informações sobre estes assuntos até 2013 usando recursos da bibliometria. Foram feitos dois tipos de análises de frequência relacionadas com os artigos de periódicos e os trabalhos do Encontro da Associação Nacional de Pesquisa em Ciência da Informação (ENANCIB): 1) níveis de produção, e 2) níveis de autoria múltipla (indicador parcial de colaboração científica). $O$ universo foram referências de 7.018 artigos de periódicos (1972-2013) e de 2.414 trabalhos de ENANCIB (1994-2013) extraídas da base ABCDM (FCl/UnB). Tais dados foram analisados no SPSS e as tabelas e gráficos foram elaborados no MS-Excel. Foi confirmada a tendência de crescimento da produção de artigos de periódicos e trabalhos de evento com patamares anuais de produção próximos de 500 artigos (2011) e cerca de 300 trabalhos de ENANCIB (2012-2013). Os gráficos sugerem a existência de correspondência sincrônica entre os dois canais medidos com prevalência de autoria múltipla nos trabalhos de ENANCIB a partir de 2005 e nos artigos de periódicos a partir de 2010.

Palavras-chaves: Bibliometria; Brasil; Ciência da Informação; comunicação científica; colaboração científica.

Scientific collaboration in the areas of information in Brazil (1972-2013)

\begin{abstract}
The collaboration between scientists is an indicator related to the scientific production and typically, both can be obtained from bibliographic databases used as information sources for decisionmaking Science, Technology \& Innovation managers, authors, editors, scientists and students. Previous studies have provided a vision of Brazilian scientific production and collaboration in the areas of information until 2010. The present study updates information about these matters until 2013 using features of Bibliometrics. Were made two types of frequency analysis related to journal articles and ENANCIB: 1) production levels, and 2) multiple authoring levels (partial indicator of scientific collaboration). The universe is composed by the 7,018 references of journal articles (1972-2013) and by 2,414 ENANCIB works (1994-2013) extracted from the ABCDM database from University of Brasília (FCI). Such data were analyzed in SPSS and the tables and charts have been prepared in MS-Excel. It was confirmed the trend of production of articles from periodicals and works with annual production levels close to 500 articles (2011) and about 300 works of ENANCIB (2012-2013). The graphics suggest the existence of synchronous correspondence between the two channels measured. There is prevalence of multiple authorship in the work of ENANCIB since 2005 and in journal articles since 2010.
\end{abstract}

Keywords: Bibliometrics; Brazil; Information Science; scientific communication; scientific collaboration. 
Resumen: La colaboración entre los investigadores es un indicador relacionado con la producción científica y en general ambos pueden obtenerse desde bases de datos bibliográficas utilizadas como fuentes de información para toma de decisiones de los administradores, autores, editores, científicos y estudiantes de Ciencia, Tecnología y Innovación. Estudios anteriores han proporcionado una visión de la producción científica brasileña y la colaboración en las áreas de información hasta 2010. El presente estudio actualiza las informaciones de estos asuntos hasta 2013 utilizando bibliometria. Se realizaron dos tipos de análisis de frecuencia relacionados con artículos de periódicos y trabajos del ENANCIB: 1) niveles de producción, 2) niveles de autoría múltiple (indicador parcial de colaboración científica). El universo fueron 7.018 referencias de artículos de revistas (1972-2013) y 2.414 obras del ENANCIB (19942013) extraídas de la base de datos ABCDM (Universidade de Brasília/FCI). Estos datos fueron analizados en SPSS y las tablas y gráficos han sido preparados en MS-Excel. Se confirmó la tendencia del crecimiento de la producción de artículos de publicaciones periódicas y trabajos, con niveles de producción anual próxima de 500 artículos de periódicos (2011) y alrededor de 300 obras de ENANCIB (2012-2013). Los gráficos sugieren la existencia de correspondencia síncrona entre los dos canales medidos y predominio de múltiple autoría en obras de ENANCIB desde 2005 y en artículos de revistas desde 2010.

Palabras-clave: Bibliometria; Brasil; Ciencia de la Información; colaboración científica; comunicación científica. 


\section{Introdução}

O processo de desenvolvimento de uma área científica inclui, entre outros fatores, o desenvolvimento da sua literatura como resultado da necessidade de registrar e disseminar os avanços científicos num processo denominado de comunicação científica. Para Meadows "a comunicação situa-se no próprio coração da ciência" sendo "tão vital quanto a própria pesquisa", pois para que os resultados de uma pesquisa sejam analisados e aceitos pelos pares, exige-se que seja necessariamente comunicada (MEADOWS, 1999, p. vii). Ele afirma ainda que as especificidades de cada área levam a adoção de maneiras diferentes de fazer e, provavelmente, de comunicar pesquisa (MEADOWS, 1999, p.39), e em relação a este aspecto, Price (1976, p. xi) vai mais além e declara que a estrutura socioeconômica de cada país influencia os indicadores científicos. Assim, o estudo da comunicação científica de uma comunidade permite a análise do seu comportamento, suas tendências, métodos, influências de pensamento, linhas e enfoques (ARBOIT, BUFREM, FREITAS, 2010, p. 19) que variam entre as diversas áreas e países.

Meadows $(1999$, p. 7) divide as atividades de comunicação em duas categorias: comunicação formal e comunicação informal. Segundo o autor a primeira categoria geralmente está disponível para um público amplo por longos períodos, enquanto a segunda geralmente é efêmera e para um público limitado. Para Targino $(2000$, p. 18) as duas formas são essenciais à evolução do conhecimento.

Durante o processo de comunicação científica uma pesquisa "costuma produzir várias publicações" que variam de formato, como os relatórios, os trabalhos de congressos, artigos de periódicos, os livros e outros, em vários suportes e com variadas funções (MUELLER, 2007, p. 22). Dois destes canais de comunicação científica serão detalhados brevemente neste trabalho: o periódico, exemplo de comunicação formal, e o evento, exemplo de comunicação informal.

Com o nascimento da ciência moderna, a necessidade de uma comunicação rápida e precisa sobre descobertas científicas desencadeou o surgimento do periódico científico (MUELLER, 2007, p. 73), bem como a exigência de um custo menor de produção pela comunidade científica (STUMPF, 1996, p. 2). São atribuídas as seguintes funções ao periódico científico (MUELLER, 1999, p. 2): estabelecer a ciência certificada, garantir um canal de comunicação entre os cientistas e de divulgação mais ampla da ciência, servir como arquivo ou memória científica, e registrar a autoria da descoberta científica. Assim, "não se pode desconsiderar a relevante contribuição registrada nos periódicos científicos" (ARBOIT; BUFREM; FREITAS, 2010, p. 26), e o estudo da comunicação científica mediada pelos 
periódicos científicos passou a ser "prática comum para explorar tendências da literatura científica e avaliar criticamente a produção de artigos nas diversas áreas do conhecimento" (BUFREM, 2006, p. 194).

O grau de importância dos periódicos científicos no Brasil pode ser avaliado observando-se os resultados de um estudo da produção de 226 bolsistas da CAPES (19952006) que apontou este canal de comunicação como o preferido para disseminar resultados de pesquisadores bolsistas das áreas de Ciências Exatas e da Terra, Ciências Biológicas, Ciências da Saúde, Ciências Agrárias, Ciências Sociais Aplicadas, Ciências Humanas e Linguística, Letras e Artes (MUELLER, 2005, p. 9-10).

O mesmo trabalho (MUELLER, 2005) apontou que apenas as Engenharias preferem os anais de eventos, e que estes foram a segunda opção nas Ciências Exatas e da Terra, Ciências Biológicas, Ciências Agrárias, Ciências Sociais Aplicadas, e Ciências Humanas. Assim, é importante ressaltar o uso do evento científico para divulgação de trabalhos, pois estes são considerados de "grande valor cultural para o desenvolvimento da área de Ciência da Informação", e meios mais informais e mais ágeis na transmissão e troca do conhecimento científico (ARBOIT, BUFREM, 2011, p. 207-208). Ainda segundo as autoras, os eventos constituem uma "fase intermediária no processo de legitimação do conhecimento científico", de forma que, "refletem a tendência das pesquisas baseada nos anseios e discussões da comunidade científica durante o evento". Permitem o contato informal de forma mais dinâmica entre os pesquisadores, congregando pessoas com interesse comum, estimulando a troca e compartilhamento de conhecimento e a criação de novas parcerias ou grupos (ARBOIT; BUFREM, 2011, p. 208, 215).

Nas áreas de informação no Brasil o maior evento científico de âmbito nacional é o Encontro Nacional de Pesquisa em Ciência da Informação (ENANCIB), promovido desde 1994 pela Associação Nacional de Pesquisa e Pós-Graduação em Ciência da Informação (ANCIB). Constitui-se em um fórum de debates e reflexões que reúne pesquisadores interessados em temas especializados da Ciência da Informação, organizados em 11 Grupos de Trabalho (GT) que foram reformulados diversas vezes desde a criação do evento (ANCIB, 2014).

As bases de dados de referências de documentos científicos são fontes que facilitam o acompanhamento da produção científica de cada área e dos seus diversos canais de comunicação. Entretanto, as bases internacionais de referências de documentos científicos não cobrem exaustivamente a produção brasileira, em especial nas áreas de informação (Arquivologia, a Biblioteconomia, a Ciência da Informação, a Documentação e a Museologia). A falta de cobertura exaustiva da produção brasileira pelas bases de dados internacionais 
incentivou ações das instituições brasileiras, em particular as universidades, na criação de bases de referências de documentos científicos produzidos no Brasil, entre as quais podemos destacar a SCIELO (BIREME/CNPq/FAPESP), BRAPCI (UFPR) e ABCDM (UnB). Tais fontes são citadas em diversos trabalhos sobre o assunto, alguns dos quais serão abordados a seguir.

O primeiro trabalho analisou alguns aspectos da transição dos autores das comunicações dos eventos para produtores de artigos de periódicos no Brasil e na Espanha (POBLACIÓN; NORONHA; CURRÁS, 1996), determinando algumas características destas duas comunidades científicas a partir da produção em eventos realizados entre 1978 e 1994. Em relação ao Brasil foram analisados trabalhos 291 comunicações de sete edições do Seminário Nacional de Bibliotecas Universitárias (SNBU), entretanto seu objetivo não estava relacionado com níveis gerais de produção ou colaboração, mas especificamente com a quantidade de autores transientes e permanentes de eventos e sua produção nos artigos de periódicos.

O segundo estudo usou a análise de rede social (ARS) em 1.361 artigos e trabalhos de 5 periódicos ${ }^{1}$ Qualis A (CAPES), fascículos de 1991 a 2005 disponíveis na Web, e edições do ENANCIB (1994-2003) e do CINFORM (2003-2005), para investigar o perfil dos autores na ciência da informação no Brasil. Apresentou, entre outros resultados, a concentração de artigos com autoria única e de autores transientes (PARREIRAS; SILVA; MATHEUS; BRANDÃO, 2006).

Outro estudo, com maior número de periódicos e artigos, analisou a evolução da produção e da colaboração (autoria múltipla) relacionada com 20 títulos brasileiros das áreas de Arquivologia, Biblioteconomia, Ciência da Informação e Documentação de 1972 a 2006 (VILAN FILHO; SOUZA; MUELLER, 2008). Foram usados no estudo referências de 3.706 artigos para exibir séries cronológicas de produção total e por tipo de autoria, concluindo, entre outros resultados: o crescimento da produção apresentou grandes variações entre 1989 e 1996; houve aumento gradativo de coautoria, que atingiu 49,16 \% em 2006; e foi prevista a supremacia da coautoria em 2007 ou 2008. Entretanto, este estudo não abordou trabalhos de eventos.

O estudo seguinte, menos abrangente, verificou características e tendências da autoria e da coautoria, entre os anos de 2000 a 2010, em 1.357 artigos de seis revistas da área de

\footnotetext{
1 Os periódicos foram Ciência da Informação, DatagramaZero, Encontros Bibli, Informação\&Sociedade e Perspectivas em Ciência da Informação. 
Ciência da Informação no Brasil|2 (NASCIMENTO, 2011). Apresentou, entre outros resultados, a prevalência da autoria múltipla a partir de 2006.

O estudo mais abrangente e mais recente, realizado por Carvalho (2013), relacionado com estas mesmas áreas do conhecimento, apresentou um quadro evolutivo da produção de artigos de periódicos científicos das áreas de informação e levantou características específicas da produção. A fonte usada foi a base $A B C D M$, produzida pela $\mathrm{FCl} / \mathrm{UnB}$, da qual foram selecionados os registros de periódicos brasileiros, exceto os periódicos Em Questão, Biblos e Estudos Históricos, num total de 2.647 referências de artigos publicados entre 2000 e 2010. Os dados coletados foram submetidos a uma análise quantitativa que permitiu a elaboração de tabelas e gráficos, nos quais se observa, entre outros resultados: a produção de artigos passou de 196 artigos em 2000, para 317 artigos em 2010; o número de títulos correntes de periódicos passou de 14 periódicos em 2000, para 17 periódicos em 2010; desde 2000, os percentuais de autorias do gênero feminino são hegemônicos em relação às do gênero masculino; e a partir de 2008, a autoria múltipla se tornou mais representativa do que a autoria única (51,38\% dos artigos publicados) e atingiu 56,15\% em 2010.

Assim, pode-se observar que os estudos produzidos até o momento proporcionam uma visão estratégica da produção brasileira nas áreas de informação até 2010, ou seja, ainda não há trabalhos que cubram a atividade científica a partir de 2011. Deste modo, este estudo complementa os estudos já realizados de forma a atualizar os dados até 2013.

Os objetivos específicos desta pesquisa são: descrever algumas características da produção brasileira de artigos científicos, especialmente a colaboração, publicados em periódicos e no principal evento nacional das áreas de informação; e apontar os níveis anuais de produção e colaboração até 2013 , relacionando cronologicamente a produção dos canais periódico e evento.

\section{Metodologia e Procedimentos}

Este estudo usou técnicas bibliométricas de análise de produção para descrever aspectos da comunidade cientifica brasileira das áreas de informação e, mais especificamente, características da colaboração científica.

A fonte de dados foi a base de dados $A B C D M$, criada na Faculdade de Ciência da Informação ( $\mathrm{FCl}$ ) da Universidade de Brasília (UnB) que facilita a identificação, o acesso e as

\footnotetext{
${ }^{2}$ Os periódicos foram Ciência da Informação, Perspectivas em Ciência da Informação, Informação \&Sociedade, Encontros Bibli, DatagramaZero e Informação\&Informação. 
análises bibliométricas de artigos de periódicos e trabalhos do Encontro Nacional de Pesquisa em Ciência da Informação (ENANCIB) publicados no Brasil nas áreas de informação. Tal escolha foi determinada especialmente considerando a abrangência da $A B C D M$ que inclui periódicos de Museologia. São cerca de 10.000 referências de artigos de mais de 30 periódicos científicos brasileiros e um português, publicados entre 1963 e 2014. Além destes a base inclui comunicações e pôsteres de todas as edições do ENANCIB, desde a primeira em 1994 até a última realizada em 2013. A referida base não está disponível em linha, mas apenas por solicitação direta à $\mathrm{FCl} / \mathrm{UnB}$, que encaminha sem custos os dados em vários formatos de arquivo (doc, txt, xls etc.) para qualquer pesquisador.

Assim, o universo deste estudo foi o conjunto dos artigos de 30 periódicos brasileiros ${ }^{3}$ e dos trabalhos de ENANCIB produzidos de 1972, ano em que a primeiro periódico científico brasileiro - a revista Ciência da Informação - iniciou sua coleção, até 2013, último ano de cobertura sistemática.

Importante ressaltar que nem todos os fascículos de periódicos correntes estavam representados na $A B C D M$ até a data da seleção dos registros, seja por ainda não terem sido publicados, ou por não terem sido processados pelos editores da referida base de dados, especialmente neste último ano de 2013. Os registros foram obtidos em maio de 2014 diretamente com os editores da $A B C D M$ em dois arquivos no formato txt, já com as indicações dos tipos de autoria.

As unidades de análise estatística foram: documento científico - artigos, comunicações e pôsteres - e suas autorias. As variáveis usadas em séries históricas anuais foram: número de documentos e tipo de autoria (única ou múltipla).

Após padronizações e conferências, os dados foram transferidos primeiramente para o sistema IBM SPSS Statistics, onde foram realizadas as análises de frequência, e em seguida, os resultados foram copiados para o MS-Excel onde foram elaboradas as tabelas e gráficos.

\footnotetext{
3 Os periódicos são: 1. Acervo: Revista do Arquivo Nacional; 2. Anais do Museu Histórico Nacional; 3. Arquivística.net; 4. Arquivo \& Administração (até 2007); 5. Brazilian Journal of Information Science; 6. Cadernos de Biblioteconomia; 7. Ciência da Informação; 8. Ciências em Museus; 9. DatagramaZero; 10. Encontros Bibli: Revista Eletrônica de Biblioteconomia e Cl; 11. InCID:Revista de Ciência da Informação e Documentação; 12. Informação \& Informação; 13. Informação \& Sociedade: Estudos; 14. Informare: Cadernos do Programa de Pós-Graduação em Cl; 15. Museologia e Patrimônio: Rev Eletrônica do PPG em Museologia e Patrimônio; 16. Perspectivas em Cl; 17. Perspectivas em Gestão \& Conhecimento; 18. Ponto de Acesso: Revista do Instituto de Cl da UFBA; 19. Revista ACB: Biblioteconomia em Santa Catarina; 20. Revista Brasileira de Biblioteconomia e Documentação; 21. Revista Brasileira de Museus e Museologia; 22. Revista da Escola de Biblioteconomia da UFMG; 23. Revista de Biblioteconomia \& Comunicação; 24. Revista de Biblioteconomia de Brasília; 25. Revista Digital de Biblioteconomia e Ciência da Informação; 26. Revista do Patrimônio Histórico e Artístico Nacional; 27. Revista Eletrônica Jovem Museologia; 28. Revista Ibero-Americana de Ciência da Informação; 29. Revista Museu; e 30. Transinformação.
} 


\section{Resultados}

Os resultados confirmam os índices obtidos por estudos anteriores em periódicos até 2011 e estabelece índices gerais dos ENANCIB de 1994 a 2013.

Podemos observar na Tabela 1 que a produção de artigos de periódicos brasileiros das áreas de informação continua subindo, tendo alcançado patamares médios anuais próximos a 450 artigos (2011-2012) e tendo chegado a quase 500 artigos em 2011 (493 artigos). Importante observar que não podemos considerar os dados de números de artigos de 2013, pois nem todos os fascículos foram incluídos na base $A B C D M$, e alguns ainda nem tinham sido publicados quando da obtenção dos dados (maio de 2014). Em relação às autorias múltiplas houve aumento regular a partir de 2008 quando alcançaram patamares próximos a 70\% em 2013.

O número anual de apresentações e pôsteres de ENANCIB também confirma a tendência de aumento da produção, conforme Tabela 1 e Gráfico 1, com índices médios anuais de cerca de 300 trabalhos (2012-2013) e percentuais de autoria múltipla sempre maiores que os dos artigos no mesmo período: 70,11\% (2011), 75,24\% (2012) e 82,65\% (2013). 
Tabela 1 - Produção de artigos de periódicos [de Cl/ das Al] e de trabalhos de ENANCIB

\begin{tabular}{|c|c|c|c|c|c|c|c|c|c|c|}
\hline \multirow[b]{2}{*}{ ANO } & \multicolumn{5}{|c|}{ Artigos de Periódicos } & \multicolumn{5}{|c|}{ Trabalhos de ENANCIB } \\
\hline & $\mathrm{AM}$ & $\%$ & $\mathrm{AU}$ & $\%$ & Total & $\mathrm{AM}$ & $\%$ & $\mathrm{AU}$ & $\%$ & Total \\
\hline 1972 & 4 & $11,43 \%$ & 31 & $88,57 \%$ & 35 & & & & & \\
\hline 1973 & 4 & $5,88 \%$ & 64 & $94,12 \%$ & 68 & & & & & \\
\hline 1974 & 7 & $13,73 \%$ & 44 & $86,27 \%$ & 51 & & & & & \\
\hline 1975 & 13 & $21,67 \%$ & 47 & $78,33 \%$ & 60 & & & & & \\
\hline 1976 & 6 & $12,77 \%$ & 41 & $87,23 \%$ & 47 & & & & & \\
\hline 1977 & 30 & $30,61 \%$ & 68 & $69,39 \%$ & 98 & & & & & \\
\hline 1978 & 16 & $24,62 \%$ & 49 & $75,38 \%$ & 65 & & & & & \\
\hline 1979 & 10 & $13,33 \%$ & 65 & $86,67 \%$ & 75 & & & & & \\
\hline 1980 & 22 & $32,35 \%$ & 46 & $67,65 \%$ & 68 & & & & & \\
\hline 1981 & 17 & $19,77 \%$ & 69 & $80,23 \%$ & 86 & & & & & \\
\hline 1982 & 15 & $24,19 \%$ & 47 & $75,81 \%$ & 62 & & & & & \\
\hline 1983 & 16 & $17,78 \%$ & 74 & $82,22 \%$ & 90 & & & & & \\
\hline 1984 & 13 & $18,06 \%$ & 59 & $81,94 \%$ & 72 & & & & & \\
\hline 1985 & 17 & $19,77 \%$ & 69 & $80,23 \%$ & 86 & & & & & \\
\hline 1986 & 18 & $21,43 \%$ & 66 & $78,57 \%$ & 84 & & & & & \\
\hline 1987 & 23 & $23,96 \%$ & 73 & $76,04 \%$ & 96 & & & & & \\
\hline 1988 & 19 & $22,35 \%$ & 66 & $77,65 \%$ & 85 & & & & & \\
\hline 1989 & 31 & $20,81 \%$ & 118 & $79,19 \%$ & 149 & & & & & \\
\hline 1990 & 45 & $36,59 \%$ & 78 & $63,41 \%$ & 123 & & & & & \\
\hline 1991 & 16 & $22,22 \%$ & 56 & $77,78 \%$ & 72 & & & & & \\
\hline 1992 & 17 & $24,29 \%$ & 53 & $75,71 \%$ & 70 & & & & & \\
\hline 1993 & 23 & $25,27 \%$ & 68 & $74,73 \%$ & 91 & & & & & \\
\hline 1994 & 12 & $14,29 \%$ & 72 & $85,71 \%$ & 84 & 9 & $39,13 \%$ & 14 & $60,87 \%$ & 23 \\
\hline 1995 & 25 & $20,49 \%$ & 97 & $79,51 \%$ & 122 & 14 & $25,00 \%$ & 42 & $75,00 \%$ & 56 \\
\hline 1996 & 40 & $21,86 \%$ & 143 & $78,14 \%$ & 183 & & & & & \\
\hline 1997 & 36 & $21,82 \%$ & 129 & $78,18 \%$ & 165 & 37 & $27,41 \%$ & 98 & $72,59 \%$ & 135 \\
\hline 1998 & 53 & $29,61 \%$ & 126 & $70,39 \%$ & 179 & & & & & \\
\hline 1999 & 55 & $29,57 \%$ & 131 & $70,43 \%$ & 186 & & & & & \\
\hline 2000 & 76 & $37,62 \%$ & 126 & $62,38 \%$ & 202 & 68 & $32,85 \%$ & 139 & $67,15 \%$ & 207 \\
\hline 2001 & 65 & $38,01 \%$ & 106 & $61,99 \%$ & 171 & & & & & \\
\hline 2002 & 60 & $32,97 \%$ & 122 & $67,03 \%$ & 182 & & & & & \\
\hline 2003 & 75 & $33,04 \%$ & 152 & $66,96 \%$ & 227 & 66 & $47,48 \%$ & 73 & $52,52 \%$ & 139 \\
\hline 2004 & 88 & $41,31 \%$ & 125 & $58,69 \%$ & 213 & & & & & \\
\hline 2005 & 98 & $44,34 \%$ & 123 & $55,66 \%$ & 221 & 71 & $58,20 \%$ & 51 & $41,80 \%$ & 122 \\
\hline 2006 & 140 & $44,44 \%$ & 175 & $55,56 \%$ & 315 & 73 & $68,22 \%$ & 34 & $31,78 \%$ & 107 \\
\hline 2007 & 173 & $47,66 \%$ & 190 & $52,34 \%$ & 363 & 120 & $70,18 \%$ & 51 & $29,82 \%$ & 171 \\
\hline 2008 & 166 & $46,50 \%$ & 191 & $53,50 \%$ & 357 & 108 & $72,00 \%$ & 42 & $28,00 \%$ & 150 \\
\hline 2009 & 196 & $49,75 \%$ & 198 & $50,25 \%$ & 394 & 113 & $71,07 \%$ & 46 & $28,93 \%$ & 159 \\
\hline 2010 & 227 & $55,10 \%$ & 185 & $44,90 \%$ & 412 & 181 & $71,83 \%$ & 71 & $28,17 \%$ & 252 \\
\hline 2011 & 292 & $59,23 \%$ & 201 & $40,77 \%$ & 493 & 183 & $70,11 \%$ & 78 & $29,89 \%$ & 261 \\
\hline 2012 & 287 & $64,49 \%$ & 158 & $35,51 \%$ & 445 & 237 & $75,24 \%$ & 78 & $24,76 \%$ & 315 \\
\hline 2013 & 255 & $68,73 \%$ & 116 & $31,27 \%$ & 371 & 262 & $82,65 \%$ & 55 & $17,35 \%$ & 317 \\
\hline TOTAL & 2801 & $39,91 \%$ & 4217 & $60,09 \%$ & 7018 & 1542 & $63,88 \%$ & 872 & $36,12 \%$ & 2414 \\
\hline
\end{tabular}

Fonte: Autor. Notas: são apresentados apenas dados dos anos em que foram realizados ENANCIB, onde: AM - autoria múltipla, AU - autoria única. Os dados dos artigos relativos a 2013 são preliminares. 
Gráfico 1 - Produção de artigos de periódicos [de Cl/ das Al] e de trabalhos de ENANCIB

$(1972-2013)$

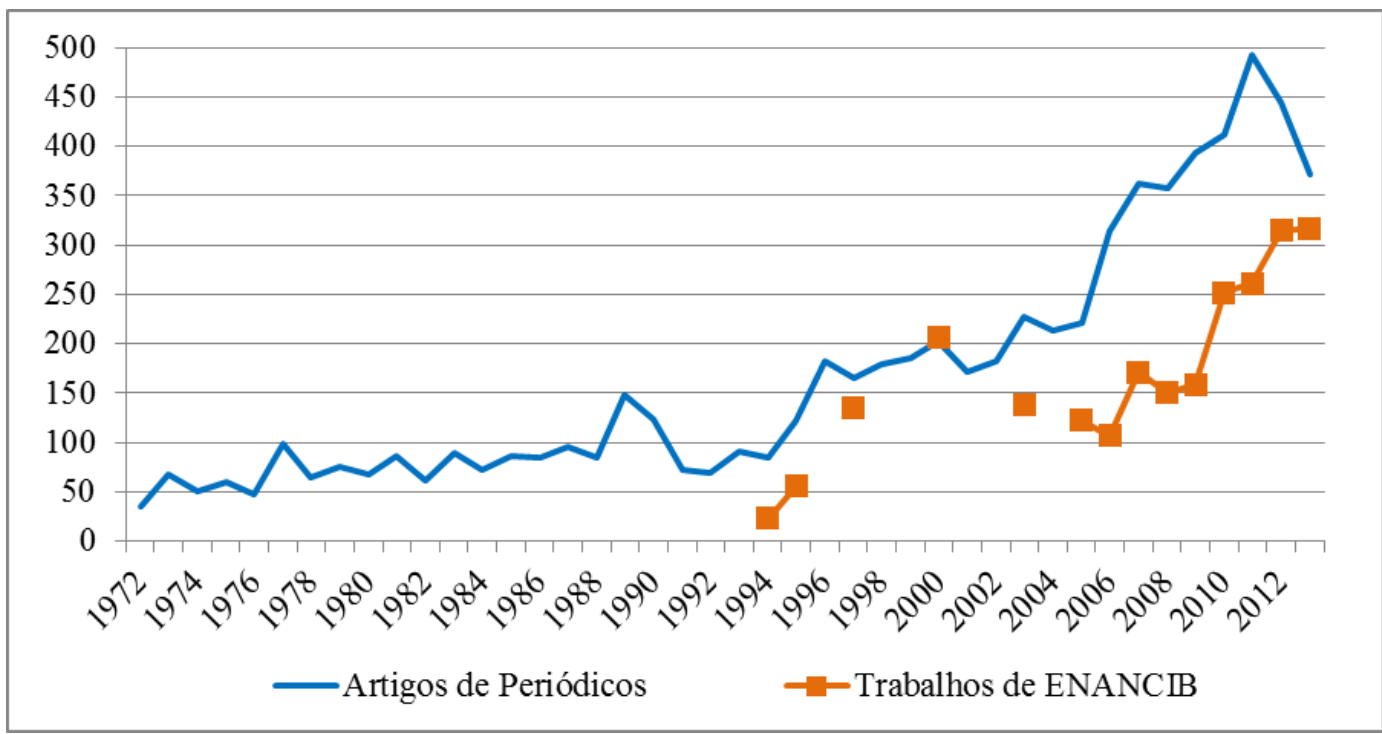

Fonte: autor. Notas: os trabalhos de ENANCIB incluem comunicações e pôsteres. Obs: entre 1996 e 2004 o ENANCIB foi realizado apenas em 1997, 2000 e 2003.

O Gráfico 1 mostra o desenvolvimento da produção de artigos de periódicos desde 1972 e de trabalhos desde 1994, ano da primeira edição do ENANCIB, oferecendo uma imagem comparativa da produção destes dois canais. Pode-se observar um crescimento mais acentuado da produção de artigos de periódicos a partir de 2005, que de quase 250 artigos chegou a quase 500 artigos em 2011. O declínio acentuado em 2012 e 2013 não deve ser considerado, pois provavelmente é decorrente de artigos ainda não processados pela base de dados ou de fascículos de periódicos em atraso. Em relação aos trabalhos de ENANCIB é visível a ausência de produção em vários anos no período entre 1995 e 2005, período em que o evento se realizou em intervalos mais longos, com a produção de trabalhos sendo praticamente a mesma de artigos em 2000 (cerca de 200 artigos e trabalhos). Entretanto, nos anos seguintes a produção de trabalhos cai para números próximos a 150 até 2010 quando sobe para níveis superiores a 250 trabalhos e atinge mais de 300 trabalhos nas duas últimas edições (2012-2013). 
Gráfico 2 - Autorias múltiplas em artigos de periódicos [de $\mathrm{Cl}$ / das Al] e em trabalhos de ENANCIB

$(1972-2013)$

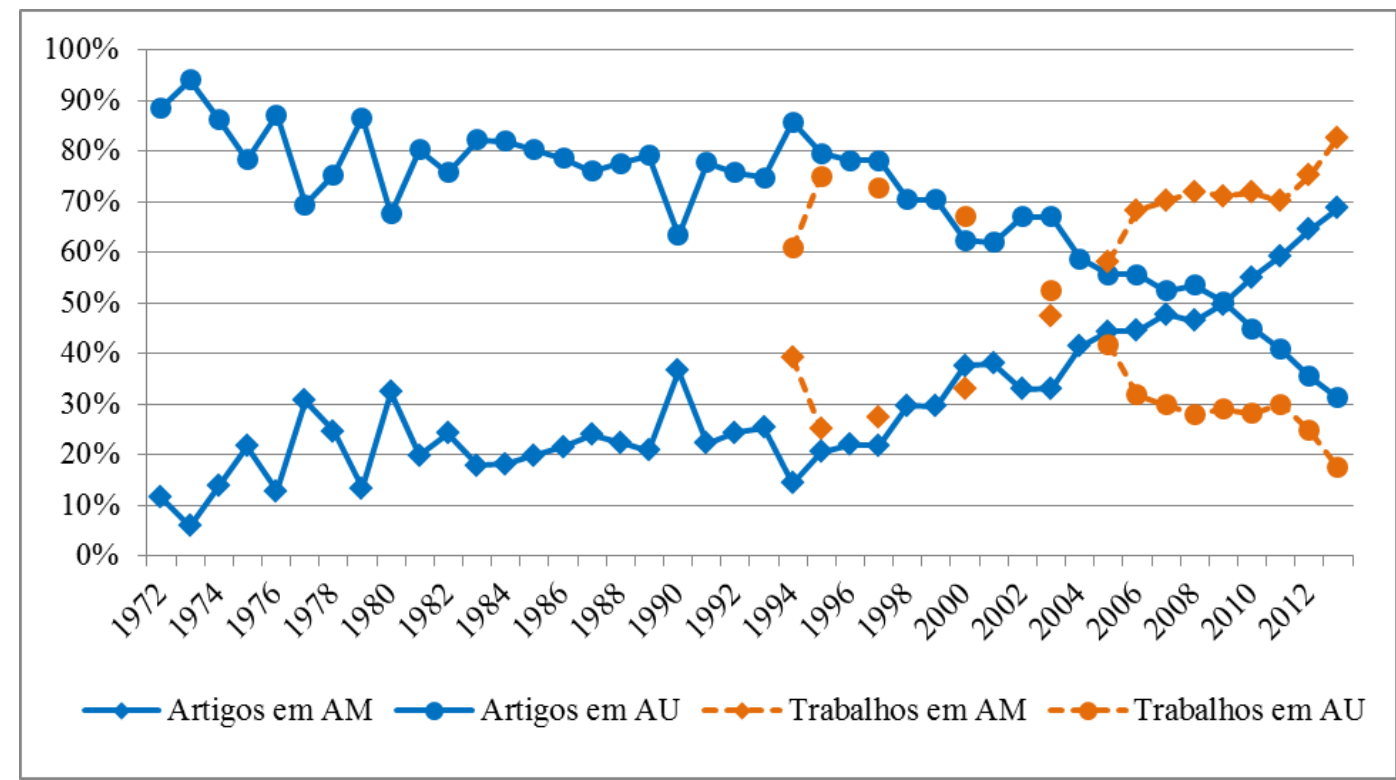

Fonte: autor. Notas: os trabalhos de ENANCIB incluem comunicações e pôsteres. Obs.: entre 1996 e 2004 o ENANCIB foi realizado apenas em 1997, 2000 e 2003.

O Gráfico 2 permite uma observação comparativa entre os percentuais de ambos os canais. Pode-se constatar que os percentuais de autoria múltipla de artigos e trabalhos são muito próximos de 1996 até o ano 2000 e a partir deste ano ambos sobem de forma diferente, alcançando índices maiores nos ENANCIB. Os índices de autoria múltipla de ENANCIB permaneceram estáveis no patamar de 70\% por seis anos consecutivos (2006-2011) e iniciam nova, e rápida, subida em 2012-2013 para atingir 82\% da autoria múltipla. Já os índices de autoria múltipla de artigos permanecem abaixo de 50\% até 2008 quando iniciam subida regular para atingir índices próximos a 70\% (68,73\%) em 2013. O fenômeno da prevalência da autoria múltipla foi observado nos artigos e nos trabalhos de ENANCIB em anos diferentes: 2005 nos trabalhos de ENANCIB e 2010 nos artigos.

\section{Conclusões}

O estudo mostrou, por meio da bibliometria, os níveis anuais de produção e colaboração científica em dois tipos de documentos das áreas de informação até 2013, complementando estudos anteriores que proporcionaram uma visão da produção científica brasileira nas áreas de informação até 2010. Foi utilizada a análise de frequência da produção de documentos (artigos de periódicos e trabalhos de ENANCIB) e dos tipos de autoria (única ou múltipla). O universo foram as referências dos artigos de periódicos de trabalhos 
(comunicações e pôsteres) publicados nos anais dos Encontros Nacionais de Pesquisa em Ciência da Informação (ENANCIB) publicados de 1994 a 2013.

Foi confirmada a tendência de crescimento da produção de artigos de periódicos e trabalhos de evento nas áreas de informação no Brasil, estabelecendo-se novos patamares anuais de produção: quase 500 artigos (2011) e pouco mais de 300 trabalhos de ENANCIB (2012-2013).

Os gráficos produzidos sugerem a existência de correspondência sincrônica entre os dois canais medidos (periódicos e ENANCIB), com prevalência de autoria múltipla alcançada primeiramente no evento e depois nos periódicos com intervalo de cinco anos. Tal fato parece lógico considerando o fluxo normal de disseminação científica na ciência em geral, pois os pesquisadores levam os resultados de suas pesquisas primeiramente aos eventos e posteriormente aos periódicos. O fenômeno observado merece estudos mais profundos para estabelecer as características desta correspondência.

Os resultados oferecem subsídios para identificar fatores contribuintes para o comportamento da produção científica brasileira nas áreas de informação.

\section{Referências}

ANCIB - ASSOCIAÇÃO NACIONAL DE PESQUISA EM CIÊNCIA DA INFORMAÇÃO. Disponível em: <www.ancib.org.br>. Acesso em: 21 jul. 2014.

ARBOIT, Aline Elis; BUFREM, Leilah Santiago. Produção de trabalhos científicos em eventos nacionais da área de ciência da informação. Transinformação, v. 23, n. 3, 2011.

ARBOIT, Aline Elis; BUFREM, Leilah Santiago; FREITAS, Juliana Lazzarotto. Configuração epistemológica da Ciência da Informação na literatura periódica brasileira por meio de análise de citações (1972-2008). Perspectivas em Ciência da Informação, v. 15, n. 1, jan./abr. 2010.

CARVALHO, Érika Rayanne Silva de. Características da produção de artigos de periódicos científicos das áreas de informação no Brasil (2000-2010). 2013. Monografia (Graduação), Universidade de Brasília, Faculdade de Ciência da Informação, 2013. 70 f.

MEADOWS, A.J. A comunicação científica. Brasília: Briquet de Lemos Livros, 1999.

MUELLER, Suzana Pinheiro Machado. O círculo vicioso que prende os periódicos nacionais. DataGramaZero: Revista de Ciência da Informação, n. 0, dez. 1999.

MUELLER, Suzana Pinheiro Machado. A publicação da ciência: áreas científicas e seus canais preferenciais. DataGramaZero: Revista de Ciência da Informação, v. 6, n. 1, fev. 2005. Artigo02. 
MUELLER, Suzana Pinheiro Machado. O periódico científico. In: CAMPELLO, Bernadete Santos; CENDÓN, Beatriz Valadares; KREMER, Jeannette M. Fontes de informação para pesquisadores e profissionais. Belo Horizonte: Editora UFMG, 2007. 319 p.

NASCIMENTO, Bruna Silva do. A Ciência da Informação no Brasil: um retrato da área através do estudo de autoria e da análise das redes de colaboração científica. 2011. Dissertação (Mestrado), Instituto de Ciência da Informação, Universidade Federal da Bahia, Salvador, 2011. $153 \mathrm{f}$.

PARREIRAS, Fernando Silva; SILVA, Antônio Braz de Oliveira e; MATHEUS, Renato Fabiano; BRANDÃO, Wladimir Cardoso. RedeCl: colaboração e produção científica em ciência da informação no Brasil. Perspectivas em Ciência da Informação, v. 11, n. 3, set./dez., 2006, p. 302-307.

POBLACIÓN, Dinah Aguiar; Noronha, DAISY Pires; CURRÁS, Emilia. Literatura cinzenta versus literatura branca: transição dos autores das comunicações dos eventos para produtores de artigos. Ciência da Informação, v. 25, n. 2, maio/ago., 1996, p. 228-242.

PRICE, Derek J. de Solla. O desenvolvimento da ciência: análise histórica, filosófica, sociológica e econômica. Rio de Janeiro: Livros Técnicos e Científicos, 1976. 98p.

STUMPF, Ida Regina Chittó. Passado e futuro das revistas científicas. Ciência da Informação, Brasília, v. 5, n. 3, 1996.

TARGINO, Maria das Graças. Comunicação científica: uma revisão de seus elementos básicos. Informação e Sociedade: Estudos, João Pessoa, v. 10, n. 2, p. 1-27, 2000.

VILAN FILHO, Jayme Leiro; SOUZA, Held Barbosa de; MUELLER, Suzana, 2008. Artigos de periódicos científicos das áreas de informação no Brasil: evolução da produção e da autoria múltipla. Perspectivas em Ciência da Informação, v.13, n. 2, maio/ago., 2008, p. 2-17.

Recebido/Recibido/Received: 2015-08-31 Aceitado/Aceptado/Accepted: 2015-10-21 